\title{
Testicular Fine-Needle Aspiration for Sperm Retrieval in Azoospermia: A Small Step toward the Technical Standardization
}

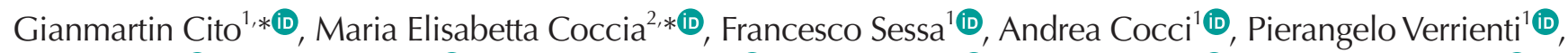

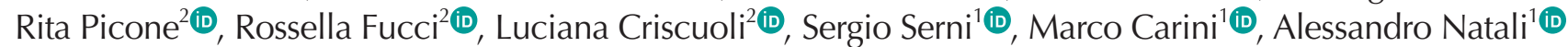 \\ ${ }^{1}$ Department of Urology, ${ }^{2}$ Assisted Reproductive Technology Center, Careggi Hospital, University of Florence, Florence, Italy
}

Purpose: The aim was to describe our preliminary experience performing testicular fine-needle aspiration (TEFNA) with a larger needle in infertile patients with obstructive azoospermia, and to provide a systematic literature review of the different testicular sperm aspiration techniques, according to the Preferred Reporting Items for Systematic Reviews and Meta-Analyses (PRISMA) statement recommendations.

Materials and Methods: We prospectively collected data between March 2017 and June 2018. All men underwent bilateral TEFNA under analgo-sedation, using a larger disposable 18-gauge butterfly needle with $60 \mathrm{~mL}$ Luer-Lock syringe attached to it.

Results: Thirty consecutive patients were enrolled. Median operative time was 16 minutes (interquartile range [IQR]: 12-30 minutes). No intraoperative complications occurred. Two/thirty patients (6.7\%) reported postoperative adverse events: 1 patient had prolonged orchialgia, 1 patient presented scrotal hematoma. Successful sperm retrieval was found in 28/30 cases (93.3\%). Median sperm concentration was $0.05 \times 10^{6} / \mathrm{mL}$ (IQR: $0.001-0.1 \times 10^{6} / \mathrm{mL}$ ). Median total sperm motility was $10 \%$ (IQR: 0\%-15\%). In 20/30 men (66.7\%) sperm retrieved was used for fresh intracytoplasmic sperm injection cycle, in 8/30 $(26.7 \%)$ sperm cryopreservation was necessary, because on the day of sperm retrieval the female resulted not responder to ovarian stimulation. In this cases mean number of 3 (IQR: 1-4) bio system straws was cryopreserved.

Conclusions: TEFNA with 18-gauge needle proved to be a feasible, safe and effective treatment, even if future prospective studies will be addressed to clarify what type of azoospermia benefits from this procedure, and if a larger needle permits to improve Assisted Reproductive Technologies (ART) outcomes.

Keywords: Azoospermia; Biopsy, fine-needle; Infertility, male; Reproductive techniques; Sperm retrieval

This is an Open Access article distributed under the terms of the Creative Commons Attribution Non-Commercial License (http://creativecommons.org/licenses/by-nc/4.0) which permits unrestricted non-commercial use, distribution, and reproduction in any medium, provided the original work is properly cited.

\section{INTRODUCTION}

Azoospermia, identified up to $15 \%$ of infertile men, recognizes a possible cause in the blockage of the male reproductive tract [1]. Thus, it can be classified as obstructive azoospermia (OA), comprising $40 \%$ of all azoospermia cases and non-obstructive azoospermia (NOA) [2]. NOA is characterized by an intrinsic, often

Received: Aug 31, 2018 Revised: Oct 23, 2018 Accepted: Oct 24, 2018 Published online Nov 27, 2018

Correspondence to: Andrea Cocci iD https://orcid.org/0000-0003-0138-6294

Department of Urology, Careggi Hospital, University of Florence, Largo Brambilla, 3, 50139, Florence, Italy.

Tel: +39-0557949203, Fax: +39-0557949046, E-mail: cocci.andrea@gmail.com

${ }^{*}$ These authors contributed equally to this work as co-first authors. 
idiopathic, testicular impairment that negatively influences spermatogenic function. Usually, open surgical testicular sperm extraction techniques are preferred in these type of patients, even if minimally invasive approaches are available in literature [2]. OA is instead usually due to ductal obstruction anywhere like efferent ducts, rete testis, ejaculatory duct, vas deferens, and epididymis. In the majority of $\mathrm{OA}$ cases, spermatogenesis is not impaired, so that successful sperm retrieval (SSR) gets to $96 \%$ to $100 \%$. In this scenario, microsurgical reconstruction might represent a safe and efficacious therapeutic option performing vasovasostomy or vasoepididymostomy when exists vasal or epidydimal obstruction, respectively. Alternatively, sperm retrieval techniques, such as testicular fine-needle aspiration (TEFNA), or testicular sperm aspiration (TESA) could be performed in order to obtain viable spermatozoa for in vitro fertilization/intracytoplasmic sperm injection (ICSI) purpose. In the assessment of azoospermic patients, TEFNA is considered effective as open biopsy, due to the high chances of sperm retrieval [3-5]. However, the quantity of testicular tissue picked is poor, depending by the method used. For this reason, previous authors described alternative techniques using larger needles [6,7] or 'biopty gun' [8], in order to improve the yield of testicular tissue, and to provide larger seminiferous tubules to the embryologist. Although the literature contains the technical details of TESA procedure, there is no general consensus on which is the best approach that allows to obtain higher SSR rates. Moreover, there is a lack of a comprehensive indication regarding to the type of azoospermia, sperm recovery rates, and specifically clinical circumstances.

The aim of this study was to describe our preliminary experience performing TEFNA with a larger needle in a case series of infertile patients with OA, and to perform a systematic review of the literature concerning the different testicular needle aspiration techniques.

\section{MATERIALS AND METHODS}

\section{Study population}

Between March 2017 and June 2018, we prospectively recruited 30 consecutive azoospermic patients who referred to our Assisted Reproductive Technologies (ARTs) Center. After the couple had undergone a complete assessment including a female gynaecological evaluation, all men underwent further assessment of the cause of azoospermia, in order to select the more appropriate sperm retrieval technique. The diagnosis of OA was based on patient history, physical and genital examination, semen analysis, endocrinology profile and genetic studies. Patients' data included information regarding age, weight and height, smoking and drinking habits, history of cryptorchidism, mumps orchitis, varicocele, environmental or radiation exposure, prescribed drug use, previous chemotherapy, or surgical procedures. All patients underwent genital examination and scrotal Doppler ultrasound scan to detect testicular volume and to exclude the presence of epididymis head or tail dilatation, unilateral or bilateral absence of vas deferens and varicocele [9]. A trans-rectal ultrasound Scan was performed to rule out the presence of prostate median cysts and of anomalies of the seminal vesicles suggestive of obstruction of the male genital tract [10]. Genetic assessment was performed, including karyotype, analysis of microdeletions for Y chromosome and mutations of Cystic Fibrosis Transmembrane Conductance Regulator (CFTR) genes. Hormonal profile was also documented during hospitalization prior to surgery in all patients. Normal levels of serum follicle-stimulating hormone (FSH), luteinizing hormone (LH), and total testosterone (TT) were considered respectively 1.5-8.0 IU/L, 1.8-12.0 IU/L, and 2.7-18.0 ng/ $\mathrm{mL}$. Prolactin levels were considered normal between 3.0 and $18 \mathrm{ng} / \mathrm{mL}$ and thyroid stimulating hormone (TSH) between 0.3 and $5.5 \mathrm{mIU} / \mathrm{L}$.

\section{Ethics statement}

All participants were adequately counselled and agreed to have their data anonymously utilized. Informed consent was submitted by all subjects when they were enrolled. The present study protocol was reviewed and approved by the Institutional Review Board (IRB) of University of Florence, Careggi Hospital (IRB No. CS/1158/07.1). All procedures were performed in accordance with the ethical standards of the institutional and national research committee and with the 1975 Helsinki Declaration.

\section{Surgical technique}

All patients enrolled underwent a bilateral TEFNA. The procedure has been performed under short-acting analgo-sedation. The scrotal skin was cleaned with $10 \%$ Povidone-iodine solution and sterile draped. Each testis 
was immobilized manually putting the epididymis and vas deferens posteriorly, safe from injury. Testes was aspirated twice at three different sites, upper, middle and lower part, using a larger disposable 18-gauge butterfly needle with $60 \mathrm{~mL}$ Luer-Lock syringe attached to it (Fig. 1). The needle was inserted into the testis to a depth of about $2 \mathrm{~cm}$. Every testicular puncture was composed of multiple precise rapid in and out movements until the yellowish fluid aspirated ceased to flow or if bloody fluid appeared. The tubing was then occluded with an artery forceps, maintaining a negative pressure. Subsequently, removing very slowly the needle from the testis and scrotal skin, we extracted some intact seminiferous tubules, protruding from the site of puncture. This tissue was prudently cut with sharp scissors and placed in the Petri dishes, using two pairs of fine tweezers. Following each puncture, the contents was washed in a 15-mL centrifuge tube containing $1 \mathrm{~mL}$ of flushing medium, using a $20 \mathrm{~mL}$ syringe filled with Hams medium. Retrieved tissue was instantaneously brought to the adjacent embryology laboratory to rule out the presence of spermatozoa. An ice pack was applied on the scrotum following the procedure for twenty minutes.

On the same day of sperm retrieval, the female underwent an oocyte pick-up procedure after ovarian stimulation protocol, in order to perform an ICSI cycle by fresh gametes.

\section{Embryological processing}

Each sample was processed by centrifugation at 1,800

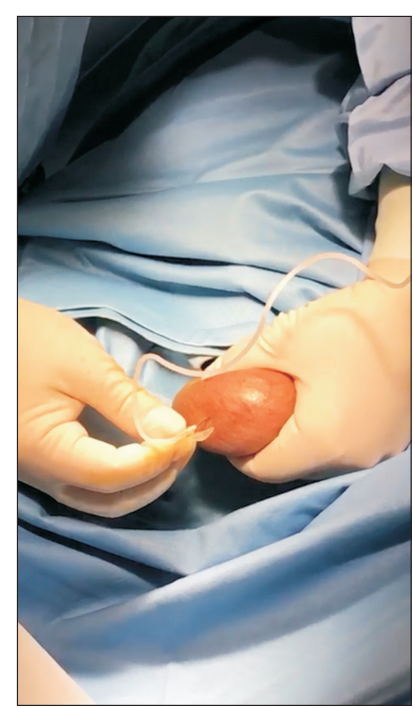

Fig. 1. Testicular fine needle aspiration. rpm for 7 minutes. The pellet was observed under inverted microscopy at $\times 200$ for a first evaluation of the presence and motility of spermatozoa.

If retrieved, spermatozoa were either used fresh for an ICSI cycle or alternatively cryopreserved for later use, if the female was not responder to ovarian stimulation protocol. If the embryologist observed more than 1-2 sperms/field (corresponding to an estimated concentration of $0.001 \times 10^{6}$ spermatozoa $/ \mathrm{mL}$ ), sperm retrieval was considered to be successful for use. For each patient the quantity and quality of sperm retrieved at the time of TEFNA was recorded, including mean sperm concentration, mean total sperm motility and mean number of bio system straws when sperm was cryopreserved.

\section{Statistical analysis}

Patient characteristics were described using medians and interquartile range (IQR) in the case of continuous variables and number and percentages for dichotomous variables. Student t-test and Mann-Whitney Utest were used to compare variables, including age and hormonal level. All collected data were evaluated with Statistical Package for Statistical Sciences (IBM SPSS ver. 22.0; IBM Co., Armonk, NY, USA).

\section{Systematic literature review}

A systematic review of English-language literature was performed up to June 2018 in accordance with the Preferred Reporting Items for Systematic Reviews and Meta-Analyses (PRISMA statement) criteria. The Medline, Scopus, Web of Science, and PubMed databases were screened separately by two different authors using a single query in order to identify the articles describing the possible techniques of TEFNA for sperm retrieval purpose in azoospermic patients. Conflicts were resolved by discussion or with an independent arbiter. The authors screened all the articles indexed in the aforementioned databases using the following query: ((((TEFNA) OR testicular fine needle aspiration) OR TESA) OR testicular sperm aspiration) OR sperm retrieval) AND azoospermia. Randomized clinical trials, retrospective, prospective, observational, and comparative studies on humans were included, while case reports and review were excluded. According to the predefined inclusion and exclusion criteria, title and abstracts were screened and articles categorized. After reading the abstract, a more thorough assess- 
ment was performed by looking at the full texts of the paper. References from the included studies were manually retrieved to identify additional studies of interest. A new excel table was built including data from the selected articles and including number of participants, interventions, comparators, outcomes, and study design (PICOS), as indicated by the Systematic Review Guidance of the Center for Reviews and Dissemination of the University of York (Center for Reviews and Dissemination. Guidance for undertaking reviews in health care. www.york.ac.uk/crd/guidance). In addition, a data extraction form was developed in order to collect, for each study, relevant information on type of azoospermia, surgical technique details, type of anesthesia, intraoperative and postoperative complications, SSR, embryological outcomes, type of ART treatment (fresh ICSI or cryopreservation). The flow chart depicting the entire review process is shown in Fig. 2.

\section{Study endpoints}

The main objectives of the study were: 1) to describe our preliminary experience using a larger needle by TEFNA in OA patients, making a small step toward the standardization of technique, 2) to provide a de- tailed overview of the available evidence on the different testicular needle aspiration techniques depicted in literature.

\section{RESULTS}

\section{Case series}

All patients had a clinical presentation of absolute azoospermia in almost two semen analyses (according to the 2010 World Health Organization guidelines). The baseline characteristics of the study cohort (30 patients) are depicted in Table 1. Median age at the time of TEFNA was 40 years (IQR: 30-51 years). No patients suffered from any chronic medical diseases, such as diabetes or cardiovascular disease. In 20/30 cases, abnormalities in CFTR gene were detected, including the more common $\Delta \mathrm{F} 508$ mutation and the rare mild mutations $2789+5 \mathrm{G}>\mathrm{A}, 2758+5 \mathrm{G}>\mathrm{A}, 2657+5 \mathrm{G}>\mathrm{A}$. The $10 / 30$ patients presented OA secondary to traumatic medullary lesion. All patients had a normal urine analysis with a negative urine-culture, as well as a negative semen culture test. Screening for sexually transmitted diseases was negative in all patients. The scrotal and trans-rectal ultrasounds documented a median

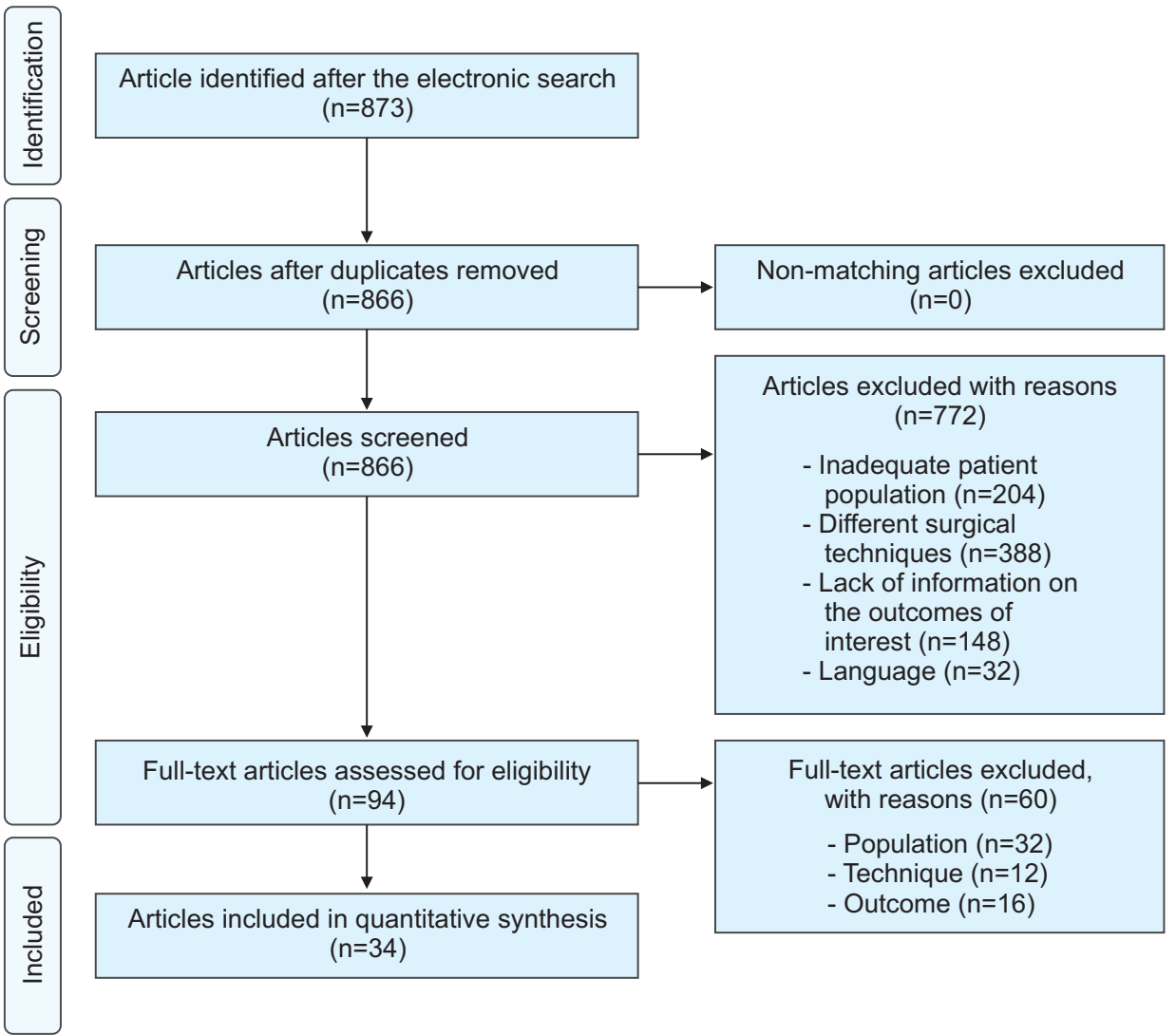

Fig. 2. Flow chart of the identified study. 
Table 1. Baseline characteristic of the study cohort

\begin{tabular}{lc}
\hline \multicolumn{1}{c}{ Parameter } & Value \\
\hline Age $(\mathrm{y})($ male) & $40(30-51)$ \\
BMI $\left(\mathrm{kg} / \mathrm{m}^{2}\right)$ & $25(18-30)$ \\
Smoker & $8(26.7)$ \\
Alcohol use & $12(40.0)$ \\
CFTR mutations & $20(66.7)$ \\
Chromosomal anomalies & $0(0)$ \\
Right TV $(\mathrm{mL})$ & $18(12-25)$ \\
Left TV $(\mathrm{mL})$ & $18(12-25)$ \\
FSH $(\mathrm{IU} / \mathrm{L})$ & $4.4(2.8-10.0)$ \\
LH $(\mathrm{IU} / \mathrm{L})$ & $3.0(2.2-4.2)$ \\
TT $(\mathrm{ng} / \mathrm{mL})$ & $4.5(1.4-9.5)$ \\
PRL $(\mathrm{ng} / \mathrm{mL})$ & $8.5(3.2-9.0)$ \\
TSH $(\mathrm{mlU} / \mathrm{L})$ & $3.1(2.2-5.5)$ \\
SSR & $28(93.3)$ \\
Sperm concentration $\left(\times 10^{6} / \mathrm{mL}\right)$ & $0.05(0.001-0.1)$ \\
Total sperm motility $(\%)$ & $10(0-15)$ \\
Straws cryopreserved & $3(1-4)$ \\
\hline
\end{tabular}

Values are presented as median (interquartile range) or number (\%). BMI: body mass index, CFTR: Cystic Fibrosis Transmembrane Conductance Regulator, TV: testicular volume, FSH: follicle stimulating hormone, LH: luteinizing hormone, TT: total testosterone, PRL: prolactin, TSH: thyroid stimulating hormone, SSR: successful sperm retrieval.

testicular right volume of $18 \mathrm{~mL}$ (IQR: $12-25 \mathrm{~mL}$ ) and a median left testicular volume of $18 \mathrm{~mL}$ (IQR: 12-25 $\mathrm{mL}$ ). Congenital bilateral absence of the vas deferens (CBAVD) was showed in 16 patients, bilateral agenesis of seminal vesicles in 4 patients. At baseline, median FSH serum level was $4.4 \mathrm{IU} / \mathrm{L}$ (IQR: 2.8-10.0 IU/L), median LH serum level was 3.0 IU/L (IQR: 2.2-4.2 IU/ L), and median TT levels were $4.5 \mathrm{ng} / \mathrm{mL}$ (IQR: 1.4-9.5 $\mathrm{ng} / \mathrm{mL}$ ). Prolactin and TSH levels were in the normal range in all patients. All men were diagnosed with OA.

Median operative time was 16 minutes (IQR: 12-30 minutes). No intraoperative complications occurred. The $2 / 30$ patients $(6.7 \%)$ reported postoperative adverse events at a median one-month follow-up: 1 patient had prolonged orchialgia for one week, 1 patient presented scrotal hematoma resolved spontaneously after few days. The overall SSR rate was $93.3 \%$ (28/30 patients). Median sperm concentration was $0.05 \times 10^{6} / \mathrm{mL}$ (IQR: $\left.0.001-0.1 \times 10^{6} / \mathrm{mL}\right)$. Median total sperm motility was 10\% (IQR: $0 \%-15 \%)$. In 20/30 men (66.7\%) sperm retrieved was used for fresh ICSI cycle, in 8/30 (26.7\%) sperm cryopreservation was necessary, because on the day of sperm retrieval the female resulted not responder to ovarian stimulation. In this cases median number of 3 (IQR: 1-4) bio system straws was cryopreserved.

\section{Evidence synthesis}

\section{1) Quality of the studies}

Overall, 873 potentially relevant articles were detected. After duplicates removed, among a total of 866 studies 94 were assessed for eligibility and finally 34 articles were included in this systematic review. Ten articles presented a prospective study design with a small study cohort sample size [11-21]. Fig. 2 outlines the study selection process according to the PRISMA flow. Table 2 provides the summary of all included studies.

\section{2) Characteristics of study population}

A total of 4,954 patients were recruited in this review. In 3,868 cases the type of azoospermia was specified. 2,343/3,868 patients (60.6\%) had a clinical presentation of NOA, 1,525/3,868 had OA (39.4\%). Of all, 4,376 men underwent testicular aspiration techniques for sperm retrieval, including TEFNA, TESA, and percutaneous testicular biopsy. Mean overall SSR rate was $74.2 \% \pm 28.9 \%$ (range: $11 \%-100 \%$ ). Mean sperm concentration and sperm motility were reported in few studies, ranging from $0.0005 \times 10^{6} / \mathrm{mL}$ to $45 \times 10^{3} / \mathrm{mL}$, and $0 \%$ to $20 \%$, respectively [11,13,20,22-25]. ART treatment planned included fresh ICSI or sperm cryopreservation.

\section{3) Technical details}

In most cases, the principal anaesthesiology approach was local anesthesia, including spermatic cord block [12,21-32]. Usually, this one was performed using 5-7 $\mathrm{mL}$ of $2 \%$ lidocaine injected with 23-gauge needle [33]. In other cases, general anesthesia can be performed to enable the operator to sample multiple regions of the testis, providing a very high likelihood of sperm retrieval for ICSI [11-13,15,34-36]. One author described the technique under epidural anesthesia [37].

Across all studies, gauge needle varied from 14- to 26-gauge. In the majority of studies describing the fineneedle aspiration (FNA) method, the needle employed ranged from 19- to 23-gauge. In few articles, some alternative techniques were detected, using larger disposable needles. In this cases, percutaneous aspiration biopsy using intravenous catheter 14- or 16-gauge or 'biopty gun' 14-gauge needle were described [11,28,30,38].

The number of testicular punctures that were per- 


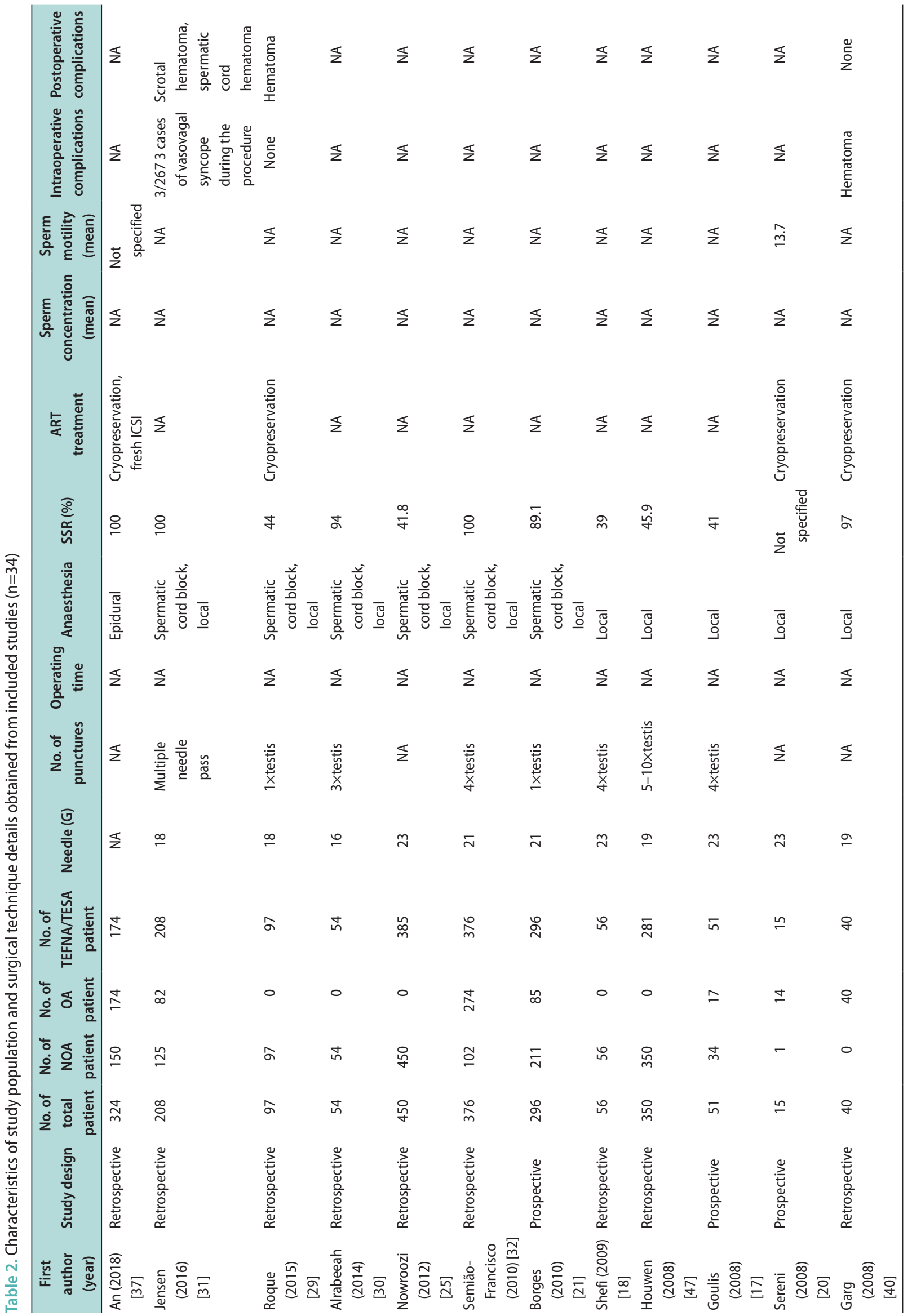




\begin{tabular}{|c|c|c|c|c|c|c|c|c|c|c|c|}
\hline \multirow{2}{*}{ 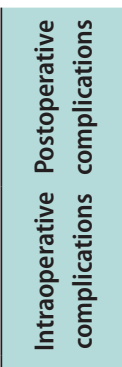 } & 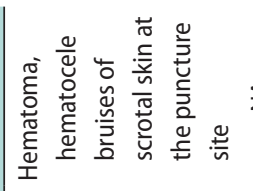 & $\Sigma$ & & \multicolumn{2}{|c|}{ 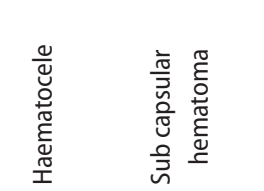 } & \multicolumn{3}{|c|}{ 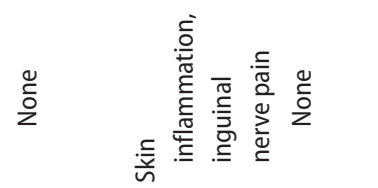 } & $\stackrel{\check{0}}{\stackrel{0}{z}}$ & $\stackrel{0}{\stackrel{0}{\circ}}$ & \multirow[t]{2}{*}{$\stackrel{0}{\tilde{O}}$} \\
\hline & 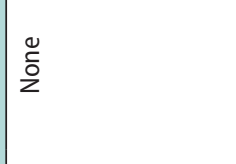 & ż & ż & 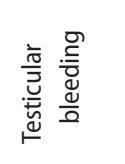 & $\stackrel{0}{\tilde{0}}$ & $\stackrel{\mathscr{c}}{\tilde{c}}$ & $\stackrel{0}{\check{0}}$ & $\stackrel{0}{\tilde{0}}$ & 气ั & $\stackrel{0}{\tilde{O}}$ & \\
\hline 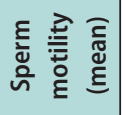 & $\Sigma$ & $m$ & 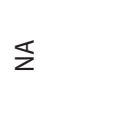 & 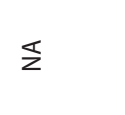 & $\stackrel{a}{a}$ & $\bar{z}$ & $\Sigma$ & $\Sigma$ & $\Sigma$ & $\Sigma$ & $\Sigma$ \\
\hline 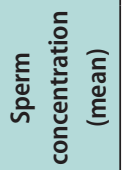 & 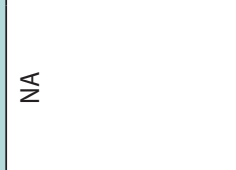 & $\Sigma$ & $\Sigma$ & $\Sigma$ & 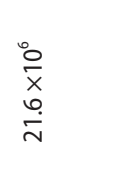 & $\Sigma$ & $\Sigma$ & $\frac{\pi}{z}$ & $\Sigma$ & $\frac{\frac{0}{0}}{\frac{0 \pi}{\frac{\pi}{5}}}$ & $\bar{s}$ \\
\hline 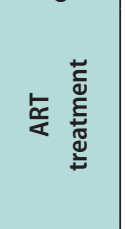 & 文 & 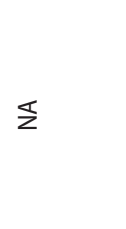 & 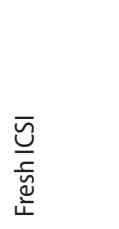 & 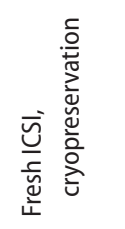 & 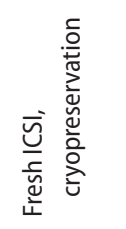 & $\bar{z}$ & 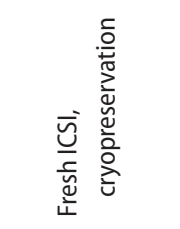 & 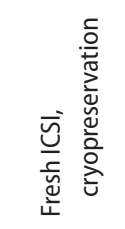 & 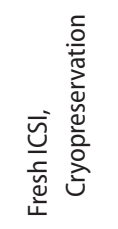 & 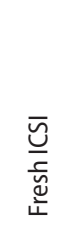 & 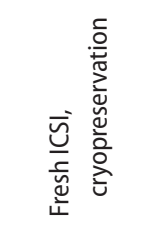 \\
\hline $\begin{array}{l}\bar{\varrho} \\
\tilde{n} \\
\tilde{n}\end{array}$ & in & $\stackrel{n}{\sim}$ & $\frac{0}{\hat{\sigma}}$ & $\stackrel{n}{\infty}$ & ¿े. & i্. & $\begin{array}{l}0 \\
\stackrel{\infty}{\circ}\end{array}$ & ○ & ส̃ & $\begin{array}{l}\infty \\
\infty \\
i \\
i n\end{array}$ & $\stackrel{\sim}{n}$ \\
\hline 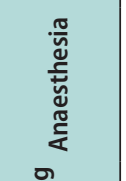 & ब্তু & $\begin{array}{l}\overline{\widetilde{N}} \\
\overline{\tilde{N}} \\
\bar{N}\end{array}$ & 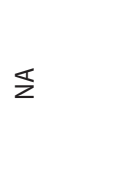 & 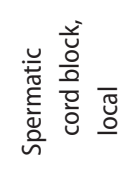 & 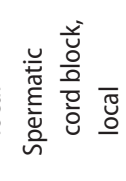 & 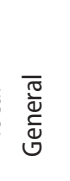 & ত্ّ & 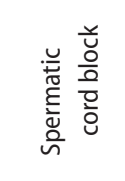 & 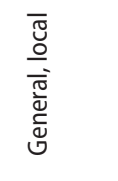 & 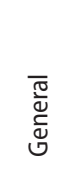 & 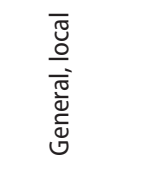 \\
\hline 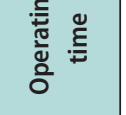 & $\Sigma$ & $\Sigma$ & $\Sigma$ & $\Sigma$ & $\S$ & $\Sigma$ & 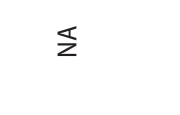 &  & $\underline{z}$ & $\S$ & $\Sigma$ \\
\hline 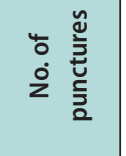 & $\begin{array}{l}n \\
\overline{\breve{u}} \\
\tilde{x} \\
0 \\
\vdots \\
0\end{array}$ & $\begin{array}{l}\stackrel{n}{\overline{\tilde{u}}} \\
\dot{x} \\
m\end{array}$ & 文 & 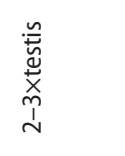 & $\underline{z}$ & $\bar{z}$ & $\Sigma$ & $\Sigma$ & 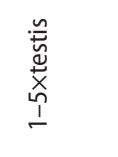 & 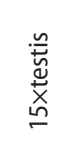 & $\begin{array}{l}\mathscr{n} \\
\overline{\tilde{u}} \\
\tilde{x} \\
0 \\
\vdots\end{array}$ \\
\hline 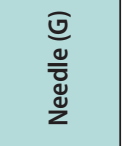 & $\approx$ & $\stackrel{\infty}{\leftarrow}$ & $\bar{\sim}$ & $\begin{array}{l}\underset{T}{N} \frac{0}{T} \\
\stackrel{T}{\sim}\end{array}$ & $\stackrel{9}{-}$ & $\approx$ & $\bar{\sim}$ & \pm & $\overline{\tilde{T}}$ & $\approx$ & $\begin{array}{c}\bar{N} \\
\alpha\end{array}$ \\
\hline 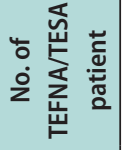 & $\stackrel{9}{\circ}$ & $\widehat{\infty}$ & ఫ্ & $\infty$ & $\stackrel{N}{N}$ & ذ్ & $\stackrel{n}{\sim}$ & $\simeq$ & 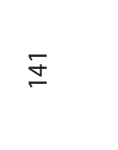 & $\stackrel{\check{\infty}}{\infty}$ & $\hat{m}$ \\
\hline 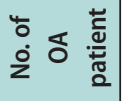 & 0 & 0 & $\stackrel{\infty}{\sim}$ & 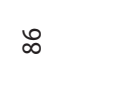 & $\stackrel{\dddot{0}}{\circ}$ & 0 & 0 & 0 & $\approx$ & $\bar{z}$ & $\bar{n}$ \\
\hline 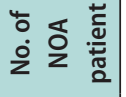 & 웅 & $\widehat{\infty}$ & ఏ & 0 & $\cong$ & ذా & $a$ & 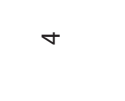 & $\stackrel{+}{m}$ & $\bar{z}$ & $\infty$ \\
\hline 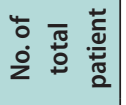 & 응 & $\widehat{\infty}$ & $\stackrel{\sim}{\sim}$ & œ & సి & ఫ & $\stackrel{n}{2}$ & $\circ$ & in & $\stackrel{\infty}{\infty}$ & $\hat{m}$ \\
\hline 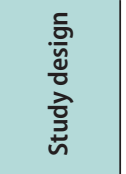 & 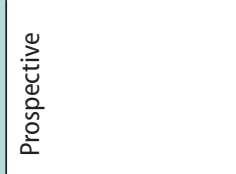 & $\bar{z}$ & 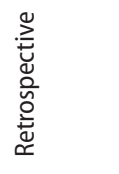 & 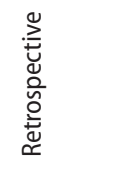 & 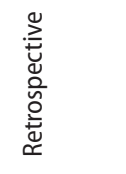 & 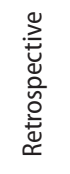 & 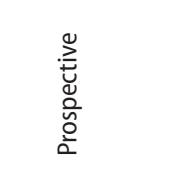 & 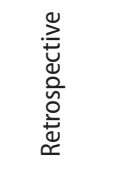 & 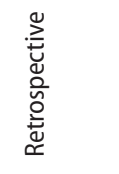 & 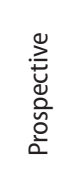 & 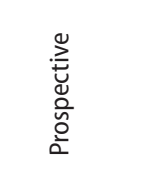 \\
\hline 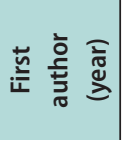 & 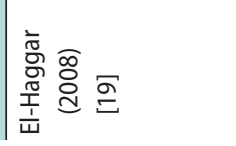 & 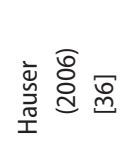 & 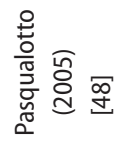 & 產离 & : & 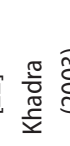 & 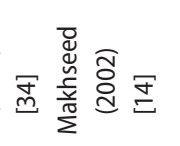 & 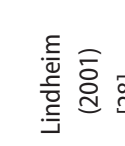 & 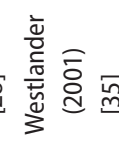 & . & 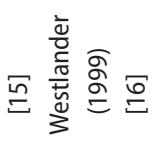 \\
\hline
\end{tabular}




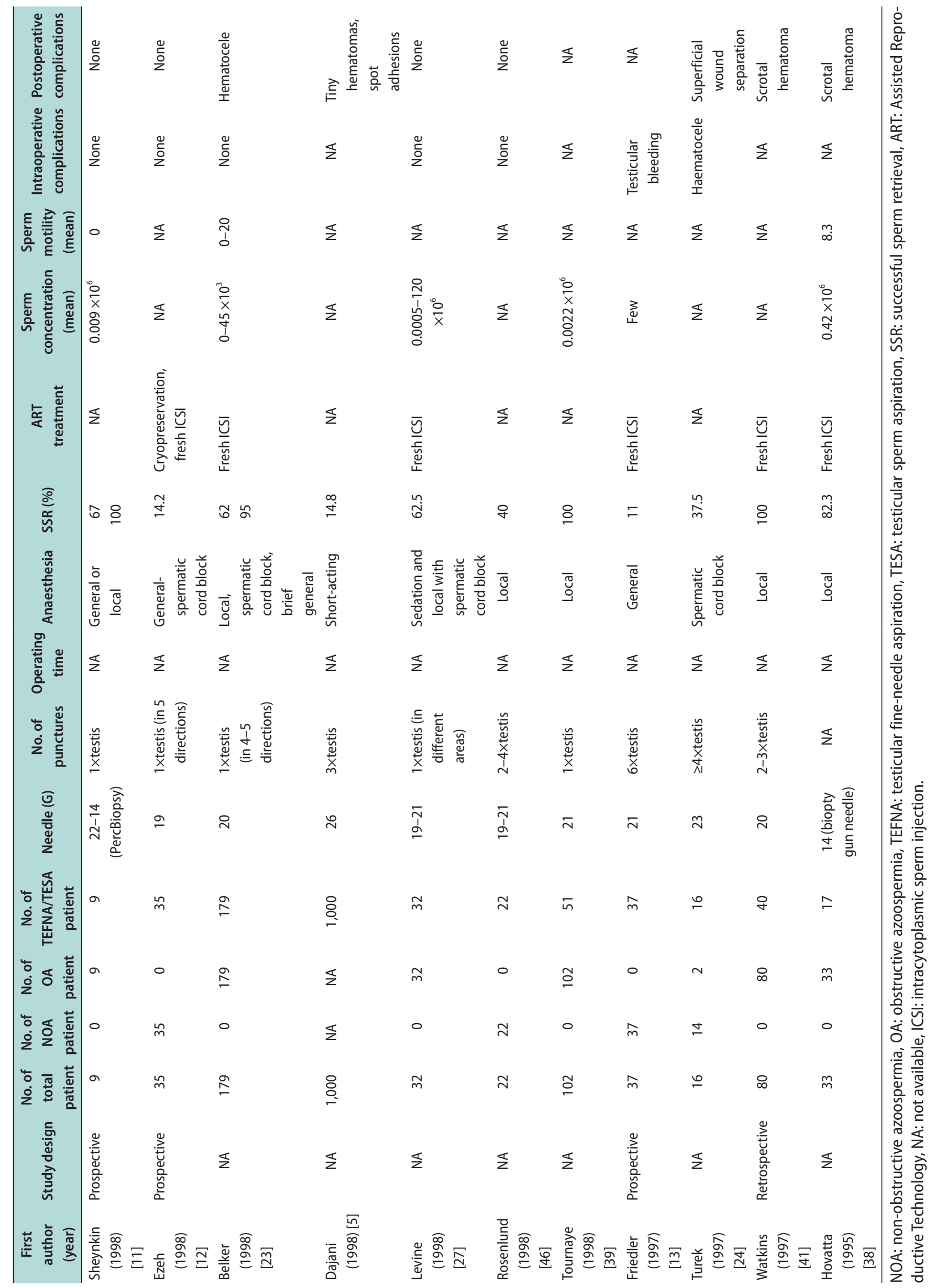


formed ranged from a minimum of 1 [12,18,21,23,27,29, $35,39]$ to a maximum of 15 [15] for each testis. Some authors preferred one single testicular puncture carried out in multiple directions (4-5) for each testis [12,23,27]. In no case, the operating time of the surgical procedure was collected.

\section{4) Intraoperative and postoperative complications}

In the majority of articles none intraoperative or postoperative complications were reported. Only few authors recorded testicular bleeding or hematocele intraoperatively [13,24,26,40]. Scrotal hematoma, superficial wound separation, spot adhesions or hematocele were the main postoperative adverse events described, even if their occurrence remains very low [5,19,22$26,29,31,41]$. In some cases, spermatic cord hematoma occurred [31]. All these postoperative complications not required surgical interventions.

\section{DISCUSSION}

The aspiration needle biopsy technique was originally described in 1930 for the diagnosis of palpable lesions [42]. Nowadays, it represents a widespread technique proposed for the exploration of many superficial organs, including thyroid nodule examination [43]. The application of testicular needle biopsy to andrology field finds a place in male infertility for ART purpose [2].

Testicular aspiration techniques (TEFNA, TESA) can be considered a relatively safe, effective, easy and inexpensive method for sperm recovery. Cost-effectiveness, simplicity and its minimal invasiveness are the main advantages, if compared with other procedures [44]. These mentioned surgical techniques can be applied both to NOA and OA patients; SSR rates are described excellent (96\% to $100 \%)$ only in OA cases, while remain lower in secretive azoospermia (10\% to $15 \%)$ [45]. According to this findings, the overall sperm retrieval rate by TEFNA or TESA has been reported different in literature, due to different surgical techniques adopted and study populations characteristics (i.e., type of azoospermia). Indeed, in the majority of the studies analysed in this review, the study population was represented by NOA patients (60\%), contrary to it was expected [46-48]. The reason why this technique is widely used in NOA can be explained by the practice of a diagnostic purpose, planned before to perform a fresh
ART treatment. Moreover, since our review comprised studies from two past decades, nowadays a diagnostic biopsy itself could be considered an obsolete procedure, not without possible risks [49]. On these bases, the SSR rate obtained in this systematic analysis, through percutaneous testicular aspiration techniques, proved to be higher (up to $100 \%$ ) in patients with OA, avoiding the need to recur to open surgical techniques. Indeed, open surgical sperm retrieval methods nowadays still remain the gold standard for secretive azoospermia, since sperm retrieval could be unsuccessful in almost $50 \%$ of cases. In these patients, especially microdissection open surgical techniques are considered the more appropriate method, because of their higher successful rates [50].

Thus, on the basis of previous evidences, in NOA patients it would be recommended to perform firstly an open testicular sperm extraction procedure, avoiding all possible complications derived from testicular aspiration [9]. According to literature, among all the patients enrolled in our case series, the SSR rate was very high (93.3\%). Indeed, all the men presented OA with marked predictive factors of positive sperm retrieval, as shown by testicular volume and serum FSH levels that were in the normal range. Nevertheless, it is known that OA patients may also have some damage in spermatogenesis, as result of the long-lasting obstruction causing impairment to the spermatogenic tubules [51].

Regarding technical details, when TEFNA or TESA was performed, needles of different sizes are generally employed, ranging from 18- to 23-gauge. Equally, the number of total testicular punctures varied considerably, achieving a maximum of 15 punctures for testis [15]. The need to use larger needles comes from the necessity to obtain more testicular tissue, in particular when a simultaneously oocyte retrieval was planned. Some authors described alternative methods, using intravenous catheter 14- or 16-gauge, in order to obtain more testicular parenchyma [26], or 18-gauge butterfly needle to examine the testis by histology $[29,31,36]$. Fahmy et al [26] described a new modification of needle aspiration biopsy, devoid of complications, simple, and inexpensive, using an ordinary intravenous catheter (14- or 16-gauge) to retrieve spermatozoa from patients with OA, comparing it to the classical FNA method. SSR rate appeared higher using an intravenous catheter compared to the standard FNA (98.1\% vs. 51.6\%, $\mathrm{p}<0.05$ ).

Also an innovative technique using a 'biopty gun' 
with 14-gauge needle, was developed by Hovatta et al [25] in cases of OA, concluding that the method used may make open scrotal operations obsolete. In several studies, TEFNA performed with relatively large needles, such as 19-gauge, reduced the necessity of open surgical biopsy [12,22,27], ranging high chances of sperm retrieval. On the other hand, the use of a smaller 26-gauge needle, as described by Dajani et al [5], provides low likelihood of sperm retrieval (SSR=14.8\%).

Therefore, on the basis of the available literature and confirmed by the results of this systematic review, no standardized needle for testicular aspiration exists. In this scenario, we preferred to use a larger needle (18-gauge) in order to ensure a very high likelihood to retrieve spermatozoa for fresh ICSI treatment. Table 3 summarized the variables of our case series compared to literature.

Regarding anaesthesiology approach, the type of anaesthesia employed during the procedure differs widely, but in most cases it is local, including the spermatic cord block or short-acting sedation. However, in order to perform more testicular punctures, a general anaesthesia could be preferred, as shown by Lewin et al [38]. Only one author performed the procedure utilizing an epidural anaesthesia [37]. In our case series, we preferred a short acting analgo-sedation, based on the short operative timing and postop recovery that allow us a rapid patient discharge.

Moreover, the needles used for TEFNA could not be large enough to allow cryopreservation of the tissue fragment. However, as shown in literature, the ability to cryopreserved spermatozoa not depends by the gauge needle utilized, but by the total number of punctures and consequently by the quantity of tissue picked. These evidences are confirmed also by our series, in which testicular aspirations allowed sperm cryopreservation when the female resulted not responder to ovar- ian stimulation.

As demonstrated by previous studies applied in the field of palpable thyroid nodules, large needle aspiration biopsy could be associated with less tissue injury than large needle cutting biopsy [43].

Nevertheless, in this systematic review, intra-operative and postoperative complication rates not differ considerably using different size of needles. In fact, scrotal hematoma occurred using a larger 'biopty gun' 14-gauge needle, as well as the smaller 26-gauge needle. 3 cases of inguinal nerve pain were described by Makhseed et al [14] using 21-gauge needle. In no case surgical interventions were necessary to repair haematocele postoperatively. Few cases of testicular bleeding and 3 cases of vasovagal syncope during the procedure under local anaesthesia were recorded [31]. Overall, in our reports, the absence of intraoperative complications and the low number of postoperative adverse events could be attributed to the choice of performing only three fine needle punctures in each testis and using ice packs following the procedure.

Regarding embryological outcomes, the majority of the studies reviewed not specified total sperm concentration and motility, but focused on the ART outcomes. The large variability in terms of sperm concentration and motility probably may depend by several factors, including the number and site of testicular punctures, and the size of the needle. In our experience, the use of 18-gauge needle enabled the embryologist to process up to $0.1 \times 10^{6} / \mathrm{mL}$ spermatozoa, with up a $15 \%$ of motility. Obtaining more motile and viable spermatozoa, could be in our opinion a key point, in order to increase the likelihood of successful ART outcomes, in terms of fertilization and cleavage rates.

Nevertheless, the current study had some limitations. The main weakness of our series is represented by the small number of the study cohort, moreover, limited to

Table 3. Mean variables of our case series compared to literature

\begin{tabular}{lll}
\multicolumn{1}{c}{ Variable } & \multicolumn{1}{c}{ Case series } & Literature review \\
\hline Gauge needle (mean) & 18 & 19.97 \\
No. of punctures (xtestis) (mean) & 3 & 3.56 \\
SSR (\%) & 93.9 & 74.26 \\
Anaesthesia type & Local, general, epidural & Short-acting \\
Intraoperative complications type & None & Bleeding, hematocele \\
Postoperative complications type & Orchialgia, hematoma & Hematoma, spot adhesions, haematocele \\
ART treatment type & Cryopreservation, fresh ICSI & Cryopreservation, fresh ICSI \\
\hline
\end{tabular}

SSR: successful sperm retrieval, ART: Assisted Reproductive Technology, ICSI: intracytoplasmic sperm injection. 
OA patients. Although SSR rate was very high (93.3\%) using a larger needle 18-gauge, we need a larger comparative studies to implement the application of this technique in clinical practice. Another possible limit of this study could be the absence of long term followup of postop complications (hematomas, fibrosis or development of antisperm antibodies) [52]. Moreover, the ICSI outcomes, including fertilization, cleavage and pregnancy rates were not recorded. On these premises, future prospective studies will be addressed to clarify what type of azoospermia benefits from this procedure, and if a larger needle permits to obtain both higher sperm concentration and more sperm motility and viability, in order to improve the overall pregnancy rate. Furthermore, future studies investigating all possible predicting factors of SSR are recommendable, with the purpose to optimize the choice of treatment.

This is the first systematic review that analyses the technical details of testicular aspiration techniques, in particular the gauge needle employed and the number of punctures performed.

The studies included in this review have several limitations including inhomogeneity with regard to study design, quality of reporting and standardization of technical surgical details. Most studies included in our review are retrospective and did not define either standardized selection criteria for the choice of a specific needle to use or the more appropriate number of testicular punctures.

\section{CONCLUSIONS}

The use of larger needles in minimally invasive testicular aspiration techniques provides to obtain more tissue, increasing the chancing of SSR, in particular when fresh ICSI cycle was planned. In our experience, the procedure using the 18-gauge needle applied twice at three different testis sites proved to be feasible, safe and effective for ART treatment. Our systematic review highlights the current lack of general consensus on the ideal study population (type of azoospermia) and on the more appropriate technical aspects (type of gauge needle, number of punctures, type of anaesthesia). For this reasons, future comparative prospective studies are mandatory to evaluate the best indication and the real contribution of TESA procedures, and to standardized this surgical technique for ART purpose.

\section{Disclosure}

The authors have no potential conflicts of interest to disclose.

\section{Author Contribution}

Conceptualization: Cito G, Coccia ME, Sessa F, Cocci A. Data curation: Cito G, Sessa F, Verrienti P. Formal analysis: Sessa F, Picone R, Fucci R. Supervision: Serni S, Carini M, Natali A. Validation: Serni S, Carini M, Natali A, Criscuoli L. Visualization: Serni S, Carini M, Natali A. Writing-original draft: Cito G, Sessa F. Writing-review \& editing: Cocci A, Natali A.

\section{REFERENCES}

1. AUA. The management of obstructive azoospermia: AUA best practice statement. Linthicum: American Urological Association Education and Research, Inc; 2010.

2. Jarow JP, Espeland MA, Lipshultz LI. Evaluation of the azoospermic patient. J Urol 1989;142:62-5.

3. Cohen MS, Frye S, Warner RS, Leiter E. Testicular needle biopsy in diagnosis of infertility. Urology 1984;24:439-42.

4. Foresta C, Varotto A, Scandellari C. Assessment of testicular cytology by fine needle aspiration as a diagnostic parameter in the evaluation of the azoospermic subject. Fertil Steril 1992;57:858-65.

5. Dajani YF, Kilani Z. Role of testicular fine needle aspiration in the diagnosis of azoospermia. Int J Androl 1998;21:295300.

6. Carpi A, Menchini Fabris F, Gorini I, Gaeta P, Romani R, Marchetti A. A percutaneous large-needle aspiration biopsy technique for histologic examination of the testis in infertile patients. Fertil Steril 1999;71:756-60.

7. Steele EK, Ellis PK, Lewis SE, McClure N. Ultrasound, antisperm antibody, and hormone profiles after testicular Trucut biopsy. Fertil Steril 2001;75:423-8.

8. Morey AF, MacDonald MF, Rozanski TA, Natarajan S, Thompson IM. Yield and efficacy of biopty gun testis needle biopsy. Urology 1999;53:604-7.

9. Ron-El R, Strauss S, Friedler S, Strassburger D, Komarovsky D, Raziel A. Serial sonography and colour flow Doppler imaging following testicular and epididymal sperm extraction. Hum Reprod 1998;13:3390-3.

10. Belenky A, Avrech OM, Bachar GN, Zuckerman Z, Ben Rafael Z, Fisch B, et al. Ultrasound-guided testicular sperm aspiration in azoospermic patients: a new sperm retrieval method for intracytoplasmic sperm injection. J Clin Ultrasound 2001; 29:339-43. 
11. Sheynkin YR, Ye Z, Menendez S, Liotta D, Veeck LL, Schlegel P. Controlled comparison of percutaneous and microsurgical sperm retrieval in men with obstructive azoospermia. Hum Reprod 1998;13:3086-9.

12. Ezeh UI, Moore HD, Cooke ID. A prospective study of multiple needle biopsies versus a single open biopsy for testicular sperm extraction in men with non-obstructive azoospermia. Hum Reprod 1998;13:3075-80.

13. Friedler S, Raziel A, Strassburger D, Soffer Y, Komarovsky $\mathrm{D}$, Ron-El R. Testicular sperm retrieval by percutaneous fine needle sperm aspiration compared with testicular sperm extraction by open biopsy in men with non-obstructive azoospermia. Hum Reprod 1997;12:1488-93.

14. Makhseed M, Al Salem MH, Ahmed MA. Percutaneous testicular sperm aspiration and intracytoplasmic sperm injection in obstructive and non-obstructive azoospermia: an easy alternative to TESE and MESA. Urol Int 2002;68:86-90.

15. Lewin A, Reubinoff B, Porat-Katz A, Weiss D, Eisenberg V, Arbel R, et al. Testicular fine needle aspiration: the alternative method for sperm retrieval in non-obstructive azoospermia. Hum Reprod 1999;14:1785-90.

16. Westlander G, Hamberger L, Hanson C, Lundin K, Nilsson L, Söderlund B, et al. Diagnostic epididymal and testicular sperm recovery and genetic aspects in azoospermic men. Hum Reprod 1999;14:118-22.

17. Goulis DG, Polychronou P, Mikos T, Grimbizis G, Gerou S, Pavlidou V, et al. Serum inhibin-B and follicle stimulating hormone as predictors of the presence of sperm in testicular fine needle aspirate in men with azoospermia. Hormones (Athens) 2008;7:140-7.

18. Shefi S, Kaplan K, Turek PJ. Analysis of spermatogenesis in non-obstructive azoospermic and virtually azoospermic men with known testicular pathology. Reprod Biomed Online 2009;18:460-4.

19. El-Haggar S, Mostafa T, Abdel Nasser T, Hany R, Abdel Hadi A. Fine needle aspiration vs. mTESE in non-obstructive azoospermia. Int J Androl 2008;31:595-601.

20. Sereni E, Bonu MA, Fava L, Sciajno R, Serrao L, Preti S, et al. Freezing spermatozoa obtained by testicular fine needle aspiration: a new technique. Reprod Biomed Online 2008;16:8995.

21. Borges E Jr, Braga DP, Bonetti TC, Pasqualotto FF, Iaconelli A Jr. Predictive factors of repeat sperm aspiration success. Urology 2010;75:87-91.

22. Levine LA, Dimitriou RJ, Fakouri B. Testicular and epididymal percutaneous sperm aspiration in men with either obstructive or nonobstructive azoospermia. Urology 2003;62: 328-32.
23. Belker AM, Sherins RJ, Dennison-Lagos L, Thorsell LP, Schulman JD. Percutaneous testicular sperm aspiration: a convenient and effective office procedure to retrieve sperm for in vitro fertilization with intracytoplasmic sperm injection. J Urol 1998;160:2058-62.

24. Turek PJ, Cha I, Ljung BM. Systematic fine-needle aspiration of the testis: correlation to biopsy and results of organ "mapping” for mature sperm in azoospermic men. Urology 1997; 49:743-8.

25. Nowroozi MR, Ahmadi H, Ayati M, Jamshidian H, Sirous A. Testicular fine-needle aspiration versus testicular open biopsy: comparable sperm retrieval rate in selected patients. Indian J Urol 2012;28:37-42.

26. Fahmy I, Kamal A, Aboulghar M, Mansour R, Serour GI, Shamloul R. Percutaneous aspiration biopsy using an intravenous catheter for testicular sperm retrieval in patients with obstructive azoospermia. Reprod Biomed Online 2004;9:1025.

27. Levine LA, Lisek EW. Successful sperm retrieval by percutaneous epididymal and testicular sperm aspiration. J Urol 1998;159:437-40.

28. Lindheim SR, Crumm K, Fisch H, Sauer MV. Testicular sperm aspiration (TESA) and its application in oocyte donation. Arch Androl 2001;46:211-5.

29. Roque M, Valle M, Marques F, Sampaio M, Geber S. Intracytoplasmic sperm injection outcomes with cryopreserved testicular sperm aspiration samples. Andrologia 2016;48:2526.

30. Alrabeeah K, Yafi F, Flageole C, Phillips S, Wachter A, Bissonnette $\mathrm{F}$, et al. Testicular sperm aspiration for nonazoospermic men: sperm retrieval and intracytoplasmic sperm injection outcomes. Urology 2014;84:1342-6.

31. Jensen CF, Ohl DA, Hiner MR, Fode M, Shah T, Smith GD, et al. Multiple needle-pass percutaneous testicular sperm aspiration as first-line treatment in azoospermic men. Andrology 2016;4:257-62.

32. Semião-Francisco L, Braga DP, Figueira Rde C, Madaschi C, Pasqualotto FF, Iaconelli A Jr, et al. Assisted reproductive technology outcomes in azoospermic men: 10 years of experience with surgical sperm retrieval. Aging Male 2010;13:44-50.

33. Li PS, Li SQ, Schlegel PN, Goldstein M. External spermatic sheath injection for vasal nerve block. Urology 1992;39:173-6.

34. Khadra AA, Abdulhadi I, Ghunain S, Kilani Z. Efficiency of percutaneous testicular sperm aspiration as a mode of sperm collection for intracytoplasmic sperm injection in nonobstructive azoospermia. J Urol 2003;169:603-5.

35. Westlander G, Rosenlund B, Söderlund B, Wood M, Bergh C. Sperm retrieval, fertilization, and pregnancy outcome in 
repeated testicular sperm aspiration. J Assist Reprod Genet 2001;18:171-7.

36. Hauser R, Yogev L, Paz G, Yavetz H, Azem F, Lessing JB, et al. Comparison of efficacy of two techniques for testicular sperm retrieval in nonobstructive azoospermia: multifocal testicular sperm extraction versus multifocal testicular sperm aspiration. J Androl 2006;27:28-33.

37. An G, Zou Z, Flannigan R, Liu J, Du H, Fu X, et al. Outcome of oocyte vitrification combined with microdissection testicular sperm extraction and aspiration for assisted reproduction in men. Med Sci Monit 2018;24:1379-86.

38. Hovatta O, Moilanen J, von Smitten K, Reima I. Testicular needle biopsy, open biopsy, epididymal aspiration and intracytoplasmic sperm injection in obstructive azoospermia. Hum Reprod 1995;10:2595-9.

39. Tournaye H, Clasen K, Aytoz A, Nagy Z, Van Steirteghem A, Devroey P. Fine needle aspiration versus open biopsy for testicular sperm recovery: a controlled study in azoospermic patients with normal spermatogenesis. Hum Reprod 1998;13: 901-4.

40. Garg T, LaRosa C, Strawn E, Robb P, Sandlow JI. Outcomes after testicular aspiration and testicular tissue cryopreservation for obstructive azoospermia and ejaculatory dysfunction. J Urol 2008;180:2577-80.

41. Watkins W, Nieto F, Bourne H, Wutthiphan B, Speirs A, Baker HW. Testicular and epididymal sperm in a microinjection program: methods of retrieval and results. Fertil Steril 1997; 67:527-35.

42. Martin HE, Ellis EB. Biopsy by needle puncture and aspiration. Ann Surg 1930;92:169-81.

43. Carpi A, Nicolini A, Marchetti C, Iervasi G, Antonelli A,
Carpi F. Percutaneous large-needle aspiration biopsy histology of palpable thyroid nodules: technical and diagnostic performance. Histopathology 2007;51:249-57.

44. Donoso P, Tournaye H, Devroey P. Which is the best sperm retrieval technique for non-obstructive azoospermia? A systematic review. Hum Reprod Update 2007;13:539-49.

45. Esteves SC, Miyaoka R, Orosz JE, Agarwal A. An update on sperm retrieval techniques for azoospermic males. Clinics (Sao Paulo) 2013;68 Suppl 1:99-110.

46. Rosenlund B, Kvist U, Plöen L, Rozell BL, Sjöblom P, Hillensjö T. A comparison between open and percutaneous needle biopsies in men with azoospermia. Hum Reprod 1998;13:126671.

47. Houwen J, Lundin K, Söderlund B, Bergh C, Kremer JA, Ekerhovd E. Efficacy of percutaneous needle aspiration and open biopsy for sperm retrieval in men with non-obstructive azoospermia. Acta Obstet Gynecol Scand 2008;87:1033-8.

48. Pasqualotto FF, Rossi LM, Guilherme P, Ortiz V, Iaconelli A Jr, Borges E Jr. Etiology-specific outcomes of intracytoplasmic sperm injection in azoospermic patients. Fertil Steril 2005; 83:606-11.

49. Schlegel PN, Su LM. Physiological consequences of testicular sperm extraction. Hum Reprod 1997;12:1688-92.

50. Chan PT, Schlegel PN. Nonobstructive azoospermia. Curr Opin Urol 2000;10:617-24.

51. Colpi GM, Pozza D. Diagnosing male infertility: new possibilities and limits. Basel: Karger; 1992;130-5.

52. Bonyadi MR, Madaen SK, Saghafi M. Effects of varicocelectomy on anti-sperm antibody in patients with varicocele. J Reprod Infertil 2013;14:73-8. 